\title{
Universal nanohydrophobicity predictions using virtual nanoparticle library
}

\author{
Wenyi Wang ${ }^{1}$, Xiliang Yan ${ }^{1,2}$, Linlin Zhao ${ }^{1}$, Daniel P. Russo ${ }^{1}$, Shenqing Wang ${ }^{2}$, Yin Liu ${ }^{3}$, Alexander Sedykh 1,4, \\ Xiaoli Zhao ${ }^{5}$, Bing Yan ${ }^{2,6}$ and Hao Zhu ${ }^{1,7,8^{*}}$
}

\begin{abstract}
To facilitate the development of new nanomaterials, especially nanomedicines, a novel computational approach was developed to precisely predict the hydrophobicity of gold nanoparticles (GNPs). The core of this study was to develop a large virtual gold nanoparticle (VGNP) library with computational nanostructure simulations. Based on the vGNP library, a nanohydrophobicity model was developed and then validated against externally synthesized and tested GNPs. This approach and resulted model is an efficient and effective universal tool to visualize and predict critical physicochemical properties of new nanomaterials before synthesis, guiding nanomaterial design.
\end{abstract}

Keywords: Nanohydrohobicity, Surface chemistry, Surface simulations, Nanomaterials design, Virtual nanoparticle library, Predictive model

Advances in nanotechnology and material sciences in the past decade have led to the rapid development of engineered nanomedicines in pharmaceutical sciences $[1,2]$. The traditional development route of new nanomaterials solely depends on experimental testing, which is costly and time consuming. With rapidly rising experimental and labor costs, computational approaches have become promising low cost alternatives to study nanomaterials [3]. To date, computational modeling approaches are broadly applied to the research and development procedure of small molecules, but rarely for larger molecules like nanomaterials [4]. This is evidenced by the many available commercial software tools [5-7] capable of predicting physicochemical properties for new druggable small molecules but none are available for new nanomedicines. Compared to small molecules, the shape, size, composition and surface ligands of nanomaterials greatly increase nanostructure complexity. Due to this increased complexity, the biological activities and therapeutic effects of nanomaterials are more difficult to model than small molecules. As a key determinant of

\footnotetext{
*Correspondence: hao.zhu99@rutgers.edu

${ }^{7}$ Department of Chemistry, Rutgers University, 315 Penn St., Camden, NJ 08102, USA

Full list of author information is available at the end of the article
}

drug pharmacokinetics, hydrophobicity influences drug solubility, absorption, distribution, and target binding characteristics, which are eventually associated with the drug efficacy, potency and toxicity $[8,9]$. Therefore, it is critical to evaluate the hydrophobicity of nanomedicines in the early stages of development, even before chemical synthesis.

In previous studies, researchers have been devoted to building quantitative structure activity relationship (QSAR) models for various bioactivities of different nanomaterials but have had limited applicability for new nanomaterial development [10-13]. Namely, two major issues limited the applicability of the resulted models: (1) the lack of enough high quality nanobioactivity data and (2) computational approaches to precisely quantify nanostructure diversity. Currently, the use of experimental values as descriptors [14, 15] prevents the predictions of new nanomaterials before chemical synthesis. On the other hand, computational calculation of descriptors allows for virtual nanoparticle generation and nano-bioactivity prediction with no chemical synthesis required. Some researchers found that descriptors calculated solely from the surface ligands of nanoparticles were useful in predicting properties. Although this is useful in predicting certain properties of nanoparticles, the effects of the

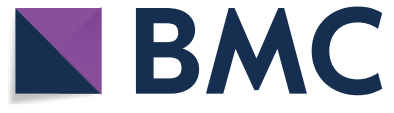

(c) The Author(s) 2019. This article is distributed under the terms of the Creative Commons Attribution 4.0 International License (http://creativecommons.org/licenses/by/4.0/), which permits unrestricted use, distribution, and reproduction in any medium, provided you give appropriate credit to the original author(s) and the source, provide a link to the Creative Commons license, and indicate if changes were made. The Creative Commons Public Domain Dedication waiver (http://creativecommons.org/ publicdomain/zero/1.0/) applies to the data made available in this article, unless otherwise stated. 
nanoparticle size and surface ligands density, position, distribution, were not considered in these studies and likely also contribute to the nano-bioactivity. More recently, however, some researchers have utilized some of these properties in addition to the general descriptor set from surface ligands, e.g., electronic properties, [16] ionic characteristics, [17] and others [18-20]. The major drawback of these available modeling studies is the lack of approaches to correctly quantify and represent nanostructure diversity during the modeling procedure. In our previous studies, we have shown that surface chemistry was the most critical factor in determining the bioactivities of gold nanoparticles (GNPs), including nanohydrophobicity [21]. Furthermore, correctly simulating surface chemistry can result in novel nanodescriptors which can be used to develop quantitative nanostructure-activity relationship (QNAR) models, showing superior advantages than traditional modeling studies [22]. Here, we report a novel approach to develop a virtual gold nanoparticle (vGNP) library with surface simulations precisely predicting nanohydrophobicity for new nanomaterials. Using this approach, a nanohydrophobicity model was developed based on surface chemistry simulation of a set of GNPs with various surface ligands. The model predictivity was further proved by experimentally synthesizing and testing nine new GNPs, and comparing their experimental/ predicted $\log \mathrm{P}$ values. The predicted nanohydrophobicity showed high correlations with experimental results, indicating the applicability of using this universal predictive modeling approach to design and select new GNPs with desired hydrophobicity.

In a recent study, we developed a novel method to construct vGNP libraries [22]. Using this approach, we constructed the vGNP library with a dataset of 41 GNPs, as shown in Fig. 1. Specifically, using the structural information of surface ligands, ligand density of each GNP, and the GNP size, the virtual structure for each of the GNPs in the library was constructed as follows. First, the gold core was constructed based on the GNP size. Then, the surface ligands, with ligand density information, were randomly attached to the gold core to simulate the experimental conditions. These 41 GNPs were synthesized and tested for their hydrophobicity. The high nanostructure diversity of these $41 \mathrm{GNPs}$, including various surface ligands, different ligand densities per GNP and various GNP sizes, and high hydrophobicity diversity (experimental $\log \mathrm{P}$ values range from -3 to 3 ) make this dataset suitable for modeling purposes. This dataset was used as the modeling set to develop nanohydrophobicity models. The experimental approaches to synthesize this GNP library and test the $\log P$ values are described in our previous study [22]. All the experimental data used to

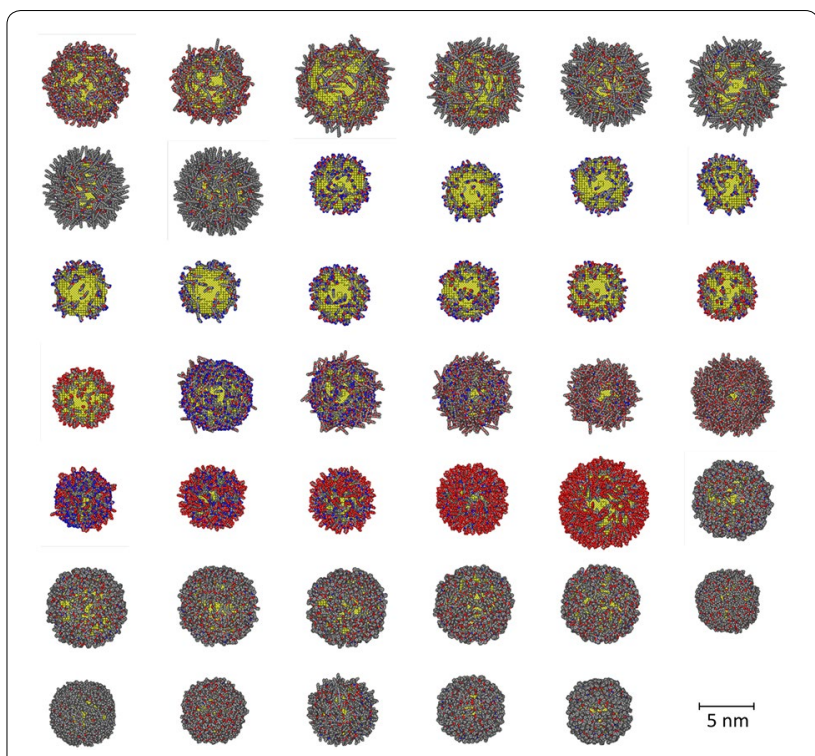

Fig. 1 The constructed vGNP library

construct the vGNP library, including the structure information of surface ligands, are provided in Additional file 4: Table SI.

Besides providing a large nanohydrophobicity dataset in this study, a new surface chemistry simulation approach was developed based on the constructed vGNP library to evaluate hydrophobicity of GNPs. The core of this technique was to evaluate the solvent accessible surface (SAS) of GNPs and to calculate the nanohydrophobicity accordingly. The SAS, also named the Connolly Surface, [23] was identified for each GNP using a grid based method [24]. The cross section (grey area) of a vGNP surface ligand was constructed in a $2 \mathrm{D}$ grid as shown in Fig. 2a. The SAS was determined by rolling a solvent probe, simulated by the size of a water molecule of radius 1.4 $\AA$, over the surface of the vGNP. Probes were placed on grid points surrounding the vGNP surface ligand. A grid point was identified as a SAS point of this vGNP when the probe was within one grid unit distance to at least one vGNP atom, and does not overlap with any other vGNP atoms [24].

Once the SAS, with all identified grid points, was constructed for a vGNP, its hydrophobicity potential was evaluated by calculating the octanol-water partition coefficient from a distance-dependent weighting function of atomic contributions $[25,26]$. The hydrophobic/hydrophilic potential of an identified SAS point was determined by nearby atoms and weighted by their distances to the SAS point. As shown in Fig. 2a, hydrophilic SAS points were colored with red while hydrophobic SAS points were colored with green. The hydrophilic/hydrophobic potential for each SAS was represented as the intensity of the corresponding 


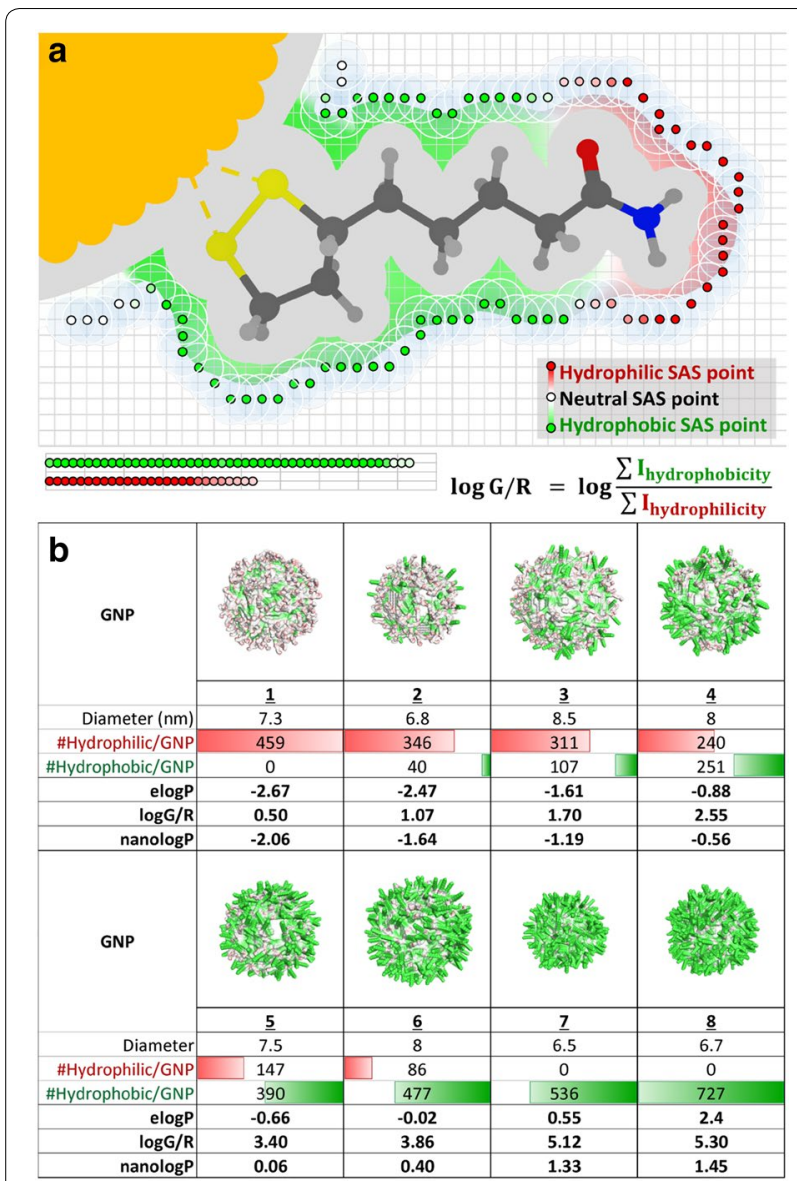

Fig. 2 Illustration of nanologP evaluations. a The SAS surface identified by rolling the solvent probe on the VGNP surface, and hydrophobicity potentials represented as colors. $\mathbf{b}$ A series of vGNPs with various calculated nanolog $P$ values

color-red as hydrophilic and green as hydrophobic. As an example, the hydrophobic potentials of eight vGNPs can be visualized in Fig. 2b. This series of GNPs were constructed with two types of surface ligands with different hydrophobicities: one ligand was hydrophilic and the other was hydrophobic. The ratio of these two types of surface ligands among the eight GNPs was gradually changed to modulate the nanohydrophobicity from low to high. From Fig. 2b, this series of GNPs showed a clear trend of hydrophobicity change with an increased ratio of hydrophilicity/hydrophobicity surface ligands. Thus, the surface colored vGNPs could be a representation of nanohydrophobicity of GNPs.

The nanohydrophobicity was then quantified using the colored vGNP. The nanohydrophobicity of a vGNP can be calculated as:

$$
\log G / R=\log \frac{\sum I_{\text {hydrophobicity }}}{\sum I_{\text {hydrophilicity }}}
$$

where $\mathrm{G}$ and $\mathrm{R}$ represent the hydrophobic potential (green) and hydrophilic potential (red) for each SAS point, and I is the intensity of hydrophobic/hydrophilic potential.

Then, with a linear regression analysis between $\log G / R$ and $\log \mathrm{P}$ values of these $41 \mathrm{GNPs}$, the following equation was generated and can be used to calculate nanologP (i.e. $\log$ P values of GNPs) values for new GNPs from their $\log G / R$ results, which were obtained from vGNP simulations:

$$
\text { nanolog } P=0.7334 * \log G / R-2.4306
$$

The calculated $\log \mathrm{P}$ values of all the 41 nanoparticles (nanologP), obtained from the above equation, were compared to their experimental $\log \mathrm{P}$ results (elogP), which were obtained by experimentally testing the partition coefficients in $\mathrm{n}$-octanol and water solutions.

The step by step instruction of vGNP generations and $\log G / R$ calculations were described in the Additional file 3 (vGNP $\log$ P Supplementary demo file) and all source code files were also shared as Python files (see details in the Additional files 1, 2, and 3).

In some previous studies, $\log \mathrm{P}$ of nanomaterials were calculated based only on surface ligand structures $[15,18$, $19,21,27]$. For comparison purposes, $\log P$ values of these 41 GNPs were calculated using four calculators, XlogP3, [28] AlogPS 2.1, [29] ClogP calculated in ChemDraw 17.0 [30] and the $\log \mathrm{P}$ model in MOE 2016 [31]. These four $\log \mathrm{P}$ calculators were built by either chemical atom/fragment contribution methods (XlogP3, $\mathrm{ClogP}$ and $\log \mathrm{P}$ in MOE) or QSAR modeling (AlogPS). These calculators are commonly used to calculate the surface ligand $\log \mathrm{P}$ and are based on various linear and non-linear modeling approaches. For example, XlogP3, AlogPS 2.1, ClogP and $\log \mathrm{P}$ in MOE were based on a nearest neighbor approach combined with linear additive model [28], associated neural networks [29], fragmental additive approach [30] and atom additive approach [31], respectively. When modeling mixtures, the weighted average according to the component fractions was used for calculating the chemical descriptors [32]. Similarly, in this study, for a GNP with two different surface ligands, its $\log \mathrm{P}$ value was calculated by averaging two ligand $\log \mathrm{P}$ values weighted by the number of the two types of ligands. As shown in Fig. 3 and Additional file 4: Table SI, the best obtained $\log \mathrm{P}$ results from commercial software, $\mathrm{X} \log \mathrm{P} 3$, which yielded a low correlation with elogP with a coefficient of determination $\left(R^{2}\right)=0.577$, and large prediction errors as Mean Absolute Error $(\mathrm{MAE})=2.633$ and root mean square error $(\mathrm{RMSE})=3.00[33,34]$. These results were much worse than that of nanologP developed in 


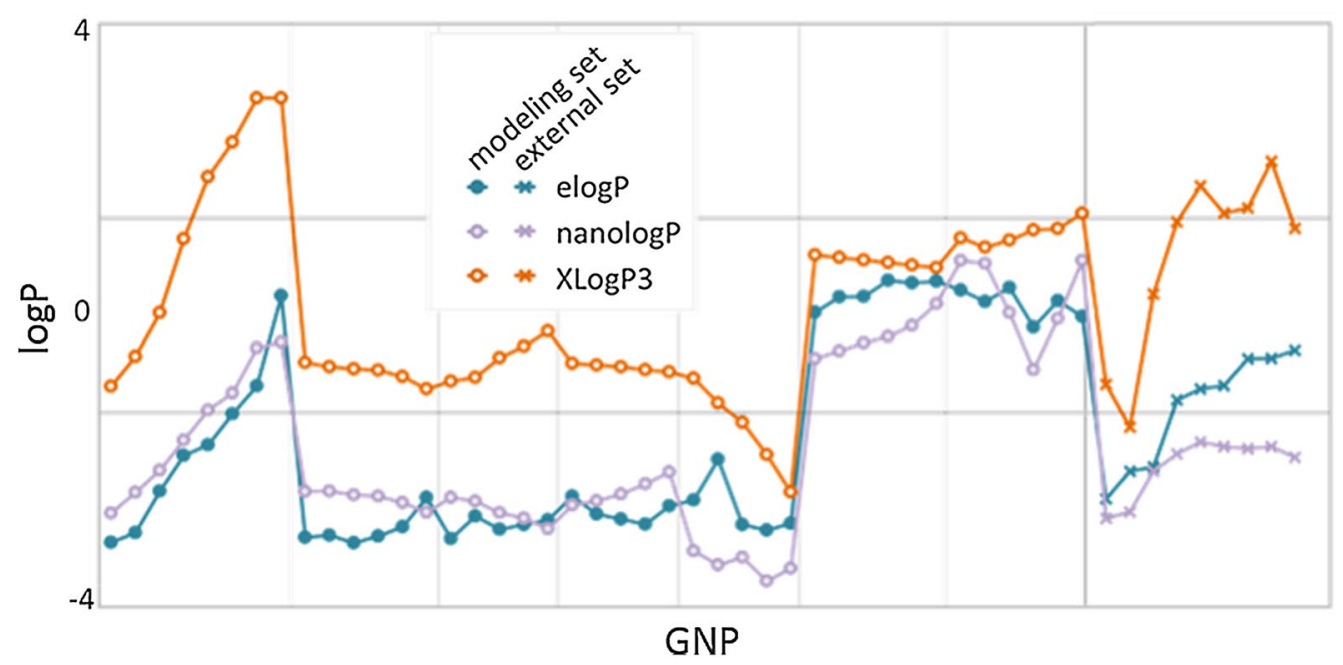

Fig. 3 Comparing the accuracy of calculated nanologP and commercial XLogP3

this study $\left(\mathrm{R}^{2}=0.884, \mathrm{MAE}=0.719\right.$ and $\left.\mathrm{RMSE}=0.81\right)$. A five-fold cross-validation was performed for nanologP and the results are similar $\left(R^{2}=0.832\right.$, MAE $=0.75$ and RMSE $=1.28$. The summary table of training and validation sets and the orginal GNP library file for calculation can be viewed in Additional files 4 and 5 .

To further validate the performance of the proposed nanologP method, we synthesized nine new GNPs with different surface ligands compared to the modeling set and experimentally obtained their elogP values. The calculated nanologP values show high predictivity for this external set with $R_{\text {ext }}^{2}=0.762, \mathrm{MAE}_{\text {ext }}=1.182$ and $\mathrm{RMSE}_{\text {ext }}=1.24$, similar to the modeling set result. In comparison, the best calculated $\log \mathrm{P}$ values from commercial software (XlogP3) show much worse prediction accuracy with $R_{\text {ext }}^{2}=0.534, \mathrm{MAE}_{\text {ext }}=3.097$ and RMSE $_{\text {ext }}=3.49$.

In this study, an applicable nanohydrophobicity computational method was developed. The results showed that precisely simulated nanostructures using the vGNP library technique was the key to the accurate calculation of physicochemical properties of GNPs, such as hydrophobicity. There is potential to adapt the approach for other nanoparticles (e.g., carbon nanotubes and silver nanoparticles). The $\log G / \mathrm{R}$ can be calculated by simulating the new type of nanoparticles with the designated core and shape, and the same hydrophobicity/hydrophilicity evaluation strategy. This is an ongoing work when more experimental data becomes available in the future. Furthermore, this approach can also be applied to the modeling and evaluation of other critical properties or bioactivities (e.g., interaction potentials with the environment, permeability through cell membranes, etc.).

\section{Additional files}

Additional file 1. Python codes for the calculation of nanologP. Additional file 2. Python codes for the calculation of nanologP. Additional file 3. A demo for calculation of nanologP. Additional file 4. Summary table of the GNP library in the training and validation sets.

Additional file 5. The original GNP library input file for the demo.

\section{Abbreviations}

GNP: gold nanoparticle; VGNP: virtual gold nanoparticle; QSAR: quantitative structure activity relationship; QNAR: quantitative nanostructure-activity relationship; SAS: solvent accessible surface.

\section{Authors' contributions}

$\mathrm{HZ}$ and WW conceived and designed the study and wrote the manuscript. $W W, X Y, L Z$ and DPR performed the three repeats of the nanologP calculation. XY calculated the logP from commercial software as control. SW and YL performed the experimental testing. AS assisted the data analysis. XZ and BY provided experimental support. All authors read and approved the final manuscript.

\section{Author details}

${ }^{1}$ The Rutgers Center for Computational and Integrative Biology, Camden, NJ 08102, USA. ${ }^{2}$ School of Chemistry and Chemical Engineering, Shandong University, Jinan 250100, China. ${ }^{3}$ Research Center for Eco-Environmental Science, Chinese Academy of Sciences, Beijing 100085, China. ${ }^{4}$ Sciome, Research Triangle Park, NC 27709, USA. ${ }^{5}$ Department of Physiological Sciences, Eastern Virginia Medical School, Norfolk, VA 23507, USA. ${ }^{6}$ School of Environment, Jinan University, Guangzhou 510632, China. ${ }^{7}$ Department of Chemistry, Rutgers University, 315 Penn St., Camden, NJ 08102, USA. ${ }^{8}$ College of Life Science and Bio-Engineering, Beijing University of Technology, Beijing 100124, China.

\section{Competing interests}

The authors declare that they have no competing interests.

\section{Availability of data and materials}

The structural information of the GNPs and the predicted nanologP values are available in Additional file 4: Table SI. The codes and the data used for calculating nanologP are available as additional files 1, 2, 5. Codes include gnprep.py, for generating vGNPs as PDB files from an SDF file; and colorQuantification. 
py, for calculating nanologP. Data include univ_multi.sdf, the original surface ligand and GNP structure of the library of 41 modeling and 9 external vGNPs.

\section{Funding}

This work was supported by the National Key R\&D Program of China (2016YFA0203103), the National Natural Science Foundation of China (91543204 and 91643204), and the Strategic Priority Research Program of the Chinese Academy of Sciences (XDB14030401).

\section{Publisher's Note}

Springer Nature remains neutral with regard to jurisdictional claims in published maps and institutional affiliations.

\section{Received: 31 May 2018 Accepted: 10 January 2019}

Published online: 18 January 2019

\section{References}

1. Shi J, Votruba AR, Farokhzad OC, Langer R (2010) Nanotechnology in drug delivery and tissue engineering: from discovery to applications. Nano Lett 10:3223-3230

2. Zhang L, Gu FX, Chan JM et al (2008) Nanoparticles in medicine: therapeutic applications and developments. Clin Pharmacol Ther 83:761-769

3. Winkler DA, Mombelli E, Pietroiusti A et al (2013) Applying quantitative structure-activity relationship approaches to nanotoxicology: current status and future potential. Toxicology 313:15-23. https://doi. org/10.1016/j.tox.2012.11.005

4. Oberdörster G (2010) Safety assessment for nanotechnology and nanomedicine: concepts of nanotoxicology. J Intern Med 267:89-105

5. Pires DEV, Blundell TL, Ascher DB (2015) pkCSM: predicting smallmolecule pharmacokinetic and toxicity properties using graph-based signatures. J Med Chem 58:4066-4072. https://doi.org/10.1021/acs.jmedc hem.5b00104

6. Hansen K, Biegler F, Ramakrishnan R et al (2015) Machine learning predictions of molecular properties: accurate many-body potentials and nonlocality in chemical space. J Phys Chem Lett 6:2326-2331. https://doi. org/10.1021/acs.jpclett.5b00831

7. Lagorce D, Sperandio O, Baell JB et al (2015) FAF-Drugs3: a web server for compound property calculation and chemical library design. Nucleic Acids Res 43:W200-W207. https://doi.org/10.1093/nar/gkv353

8. Krämer SD, Wunderli-Allenspach H (2001) Physicochemical properties in pharmacokinetic lead optimization. Farmaco 56:145-148

9. Gleeson MP (2008) Generation of a set of simple, interpretable ADMET rules of thumb. J Med Chem 51:817-834. https://doi.org/10.1021/jm701 $122 \mathrm{q}$

10. Walkey CD, Olsen JB, Song F et al (2014) Protein corona fingerprinting predicts the cellular interaction of gold and silver nanoparticles. ACS Nano 8:2439-2455. https://doi.org/10.1021/nn406018q

11. Chen R, Zhang Y, Monteiro-Riviere NA, Riviere JE (2016) Quantification of nanoparticle pesticide adsorption: computational approaches based on experimental data. Nanotoxicology 10:1118-1128. https://doi. org/10.1080/17435390.2016.1177745

12. Pathakoti K, Huang MJ, Watts JD et al (2014) Using experimental data of Escherichia coli to develop a QSAR model for predicting the photoinduced cytotoxicity of metal oxide nanoparticles. J Photochem Photobiol B Biol 130:234-240. https://doi.org/10.1016/j.jphotobiol.2013.11.023

13. Fourches D, Pu D, Tassa C et al (2010) Quantitative nanostructure - activity relationship modeling. ACS Nano 4:5703-5712. https://doi. org/10.1021/nn1013484

14. Mikolajczyk A, Malankowska A, Nowaczyk G et al (2016) Combined experimental and computational approach to developing efficient photocatalysts based on $\mathrm{Au} / \mathrm{Pd}-\mathrm{TiO}_{2}$ nanoparticles. Environ Sci Nano 3:1425-1435. https://doi.org/10.1039/c6en00232c
15. Fourches D, Pu D, Li L et al (2016) Computer-aided design of carbon nanotubes with the desired bioactivity and safety profiles. Nanotoxicology 10:374-383. https://doi.org/10.3109/17435390.2015.1073397

16. Jagiello K, Chomicz B, Avramopoulos A et al (2017) Size-dependent electronic properties of nanomaterials: How this novel class of nanodescriptors supposed to be calculated? Struct Chem 28:635-643. https://doi. org/10.1007/s11224-016-0838-2

17. Sizochenko N, Mikolajczyk A, Jagiello K et al (2018) How the toxicity of nanomaterials towards different species could be simultaneously evaluated: a novel multi-nano-read-across approach. Nanoscale 10:582-591. https://doi.org/10.1039/c7nr05618d

18. Toropov AA, Toropova AP, Puzyn T et al (2013) QSAR as a random event: modeling of nanoparticles uptake in $\mathrm{PaCa} 2$ cancer cells. Chemosphere 92:31-37. https://doi.org/10.1016/j.chemosphere.2013.03.012

19. Luan F, Tang L, Zhang L et al (2016) A further development of the QNAR model to predict the cellular uptake of nanoparticles by pancreatic cancer cells. Food Chem Toxicol 112:571-580

20. Mikolajczyk A, Pinto HP, Gajewicz A et al (2015) Ab initio studies of anatase $\mathrm{TiO}_{2}$ (101) surface-supported Au8 clusters. Curr Top Med Chem 15:1859-1867. https://doi.org/10.2174/1568026615666150506151826

21. Li S, Zhai S, Liu Y et al (2015) Experimental modulation and computational model of nano-hydrophobicity. Biomaterials 52:312-317. https:// doi.org/10.1016/j.biomaterials.2015.02.043

22. Wang W, Sedykh A, Sun H et al (2017) Predicting nano-bio interactions by integrating nanoparticle libraries and quantitative nanostructure activity relationship modeling. ACS Nano. https://doi.org/10.1021/acsnano.7b070 93

23. Connolly M (1983) Solvent-accessible surfaces of proteins and nucleic acids. Science 221:709-713. https://doi.org/10.1126/science.6879170

24. Sethian JA (1998) Fast marching methods and level set methods for propagating interfaces. Proc Natl Acad Sci USA 93(4):1591-1595

25. Wildman SA, Crippen GM (1999) Prediction of physicochemical parameters by atomic contributions. J Chem Inf Comput Sci 39:868-873. https ://doi.org/10.1021/ci990307|

26. Heiden W, Moeckel G, Brickmann J (1993) A new approach to analysis and display of local lipophilicity/hydrophilicity mapped on molecular surfaces. J Comput Aided Mol Des 7:503-514. https://doi.org/10.1007/ BF00124359

27. Moyano DF, Goldsmith M, Solfiell DJ et al (2012) Nanoparticle hydrophobicity dictates immune response. J Am Chem Soc 134:3965-3967. https ://doi.org/10.1021/ja2108905

28. Cheng T, Zhao Y, Li X et al (2007) Computation of octanol-water partition coefficients by guiding an additive model with knowledge. J Chem Inf Model 47:2140-2148. https://doi.org/10.1021/ci700257y

29. Tetko IV, Tanchuk VY (2002) Application of associative neural networks for prediction of lipophilicity in ALOGPS 2.1 program. J Chem Inf Comput Sci 42:1136-1145. https://doi.org/10.1021/ci025515j

30. BioByte. http://www.biobyte.com/. Accessed 22 Feb 2018

31. Chemical Computing Group ULC (2013) Molecular operating environment (MOE)

32. Muratov EN, Varlamova EV, Artemenko AG et al (2012) Existing and developing approaches for QSAR analysis of mixtures. Mol Inform 31:202-221. https://doi.org/10.1002/minf.201100129

33. Sizochenko N, Jagiello K, Leszczynski J, Puzyn T (2015) How the "liquid drop" approach could be efficiently applied for quantitative structureproperty relationship modeling of nanofluids. J Phys Chem C 119:2554225547. https://doi.org/10.1021/acs.jpcc.5b05759

34. Mikolajczyk A, Sizochenko N, Mulkiewicz E et al (2017) Evaluating the toxicity of $\mathrm{TiO}_{2}$-based nanoparticles to Chinese hamster ovary cells and Escherichia coli: a complementary experimental and computational approach. Beilstein J Nanotechnol 8:2171-2180. https://doi.org/10.3762/ bjnano.8.216 